\title{
Investigations of free space and deep space optical communication scenarios
}

\author{
Shun-Ping Chen ${ }^{1,2}$ (1)
}

Received: 29 April 2021 / Revised: 21 August 2021 / Accepted: 27 August 2021 / Published online: 13 September 2021

(C) The Author(s) 2021

\begin{abstract}
The link performance of free space optical communications (FSOC) and deep space optical communications (DSOC) are investigated by considering two scenarios in space communications, for example, for the downlink and uplink between the earth ground stations and the near earth geostationary (GEO) satellites, and between the earth and spacecraft with large distance of 1 astronomical unit (AU) to the earth. Generally a distance larger than $0.01 \mathrm{AU}$ or approximately 1,500,000 km from Earth is considered as deep space. In these theoretical investigations, different realistic system parameters for the optical lasers, transmitters, receivers, avalanche photodiodes (APDs), optical telescopes, atmospheric disturbances like scintillation and absorption are considered. The simulation results are compared with existing project data and valuable ESA experimental results to verify and improve the simulation models. The comparison in this paper shows that the simulation models for the link budget and the scintillation estimation are feasible for the investigations of FSOC and DSOC, and can be used to investigate improved design and implementation of DSOC projects for planned long-term and medium-term space missions.
\end{abstract}

Keywords Free space optical communications (FSOC) - Deep space optical communications (DSOC) - Geostationary orbit (GEO) - Low earth orbit (LEO) satellites · Link budget · Scintillation · Beam wander · Absorption · Scattering · Laser · Avalanche photodiode (APD) $\cdot$ Telescope $\cdot$ Uplink $\cdot$ Downlink $\cdot$ Sun-earth-probe angle (SEP Angle)

\section{Introduction}

Laser optical communication links show significant advantages in comparison with classical radio frequency microwave links for space communication technologies, in terms of link performance, system size, and achievable transmission bitrates. Many projects have been proposed by the member agencies of the International Astronautical Federation (IAF) like NASA, ESA etc. and trials have been successfully carried out in the last decades. The link performance of free space optical communications (FSOC) and deep space optical communications (DSOC) are investigated by considering different scenarios in space communications. For example, for the downlink and uplink between

Shun-Ping Chen

shun-ping.chen@h-da.de

1 Visiting Scientist at ESA/ESOC, Robert-Bosch-Strasse 5, 64293 Darmstadt, Germany

2 Institute of Communication Technologies, Darmstadt University of Applied Sciences, Birkenweg 8, 64295 Darmstadt, Germany
Earth ground stations and (a) satellites in low Earth orbit or geostationary satellites, or (b) spacecrafts in the lunar orbit, or (c) spacecrafts with large distances of about 1-3 AU to Earth. In these theoretical investigations, different realistic system parameters for the optical lasers, transmitters, receivers, avalanche photodiodes (APDs), optical telescopes, atmospheric disturbances like scintillation and absorption are considered. For extremely large distances the robust pulse position modulation scheme (PPM) and APD photon counting receivers help to achieve the desired transmission bitrates. Various results like transmitter and receiver power densities, laser beam spot sizes, and received power at the receiver are simulated. The results are compared with some existing project planning data and experimental results to verify and improve the simulation model. The investigation results can be utilized in the design and implementation of the DSOC projects for planned long-term and medium-term space missions. 


\section{Theoretical background}

Compared with the classical radio frequency deep space communications in S, X, and Ka band, deep space optical communications show advantages in terms of beam divergence (improvement by a factor of 100), high bitrate delivery with significantly reduced aperture size of the flight terminal [1]. The demonstration and measurement projects NASA LLCD (Lunar Laser Communication Demonstration), ESA ARTEMIS SILEX (Semiconductor-laser Intersatellite Link EXperiment) and ARTEMIS to OGS (Optical Ground Station) have been carried out, also with cooperation between NASA and ESA [2-4]. Many suggestions concerning the efficient transmitter telescope, APD photon counting receiver system [5], and PPM modulation scheme have been proposed to achieve the optimum system performance due to extremely large distances in the case of deep space optical communications. Also future projects are being planned like PSYCHE [6] and DOCS L5 [7].

For the general link budget calculation, in free space optical communications, plane wave approximation, spherical wave propagation, and Gaussian beam propagation models are commonly used $[8,9]$. We focus on the spherical wave propagation and Gaussian beam propagation. The latter two methods will deliver identical results, if the same planning parameters are used.

\subsection{Spherical beam propagation}

The link budget calculation of the spherical wave propagation is

$P_{R}(L)=P_{T} \cdot \eta_{T} \cdot g_{T} \cdot \eta_{A t m}(L) \cdot \eta_{F S}(L) \cdot g_{R} \cdot \eta_{P} \cdot \eta_{R}$

with $P_{T}, P_{R}(L)$ as transmitted power and received power at a distance $L ; \eta_{T}, \eta_{R}$ as transmitter and receiver telescope aperture efficiencies; $\eta_{P}$ as pointing efficiency; $g_{T}, g_{R}$ as transmitter and receiver aperture gain; $\eta_{A t m}(L)$ as the atmospheric transmittance due to absorption loss, Rayleigh scattering (size of the scatterers $\ll \lambda$ ) and Mie scattering (size of the scatterers $\approx \lambda) ; \eta_{F S}(L)$ as free space propagation efficiency, corresponding to the beam divergence.

\subsection{Gaussian beam propagation}

Alternatively the Gaussian beam propagation model uses the beam radius or half spot size $w_{0}$ and $w(L)$ to describe the wave front. Due to the divergence, the beam radius increases with distance $L$ from the transmitter. The free space transmission efficiency $\eta_{F S}(L)$ of the link budget will be basically replaced by $w(L)^{2} / w_{0}^{2}$ representing the beam divergence. The distribution of the irradiance $I(r, L)$ of the Gaussian beam with the beam radius $w(L)$ at a distance $L$ from the transmitter, with $r$ as lateral radius from the beam axis, $L_{R}$ as Rayleigh length, and $D_{R}$ as receiver aperture diameter, can then be written as

$\frac{I(r, L)}{I(r, 0)}=\frac{w_{0}^{2}}{w(L)^{2}} \cdot \exp \left(-\frac{2 \cdot r^{2}}{w(L)^{2}}\right) \cdot \eta_{T} \cdot \eta_{A t m}(L) \cdot \eta_{P}$

$w(L)=w_{0} \cdot \sqrt{1+\left(\frac{L}{L_{R}}\right)^{2}}$

$L_{R}=\frac{\pi \cdot w_{0}^{2}}{\lambda}$

$P(r, L)=I(r, L) \cdot \pi \cdot D_{R}^{2} \cdot \eta_{R}$

\subsection{Transmitter and receiver}

Coherent lasers for free space optical communications are either high power solid state lasers like Ti:Sapphire lasers pumped by an argon laser, or a classical commercial III/V semicondutor InGaAsP laser diode (approximately $10 \mathrm{~mW}$ ). In combination with laser diodes, also EDFAs (Erbium Doped Fiber Amplifiers) can be used to achieve approximately $25 \mathrm{~dB}$ gain.

The silicon photodiode or the InGaAsP photodiode, also used in fiber optical communication networks, can be used for FSOC. For the extreme distance of 1-3 AU (astronomical unit, approximately $150,000,000-450,000,000 \mathrm{~km}$ ) only few photons per pulse will arrive at the receiver. For this case an avalanche photodiode (APD) with built-in amplification in the Geiger mode can be used, as single photon counter, to increase the receiver responsivity significantly. However due to the deadtime of the APD in Geiger mode, the slot width of the PPM symbols cannot be chosen arbitrarily small, so that the total bitrate is also limited.

\subsection{Telescopes}

At the transmitter, the laser beam will be collimated to the telescope aperture. Due to the Gaussian distribution of the fundamental mode $T E M_{00}$, the aperture is never illuminated $100 \%$ perfectly, leading to the above mentioned transmitter efficiency of approximately $\eta_{T} \approx 70 \%$. Depending on the size of the aperture, a corresponding gain can be achieved. Due to the limited space on a spacecraft or satellite, the aperture size will be limited. On the other hand, the ground station telescope can be designed large enough to achieve the best received beam power and the best possible signalto-noise ratio (SNR). 
In general, the received noise is caused by the blue sky background, the earth background, the sun light or the irradiance from other stars or planets.

\subsection{Absorption and scattering in the atmosphere}

During the free space optical wave propagation through the atmosphere, some photons will be absorbed by molecules and electrons in the atmosphere. The absorption by molecules is in the infrared spectral range, whereas the absorption by electrons is mostly in the ultra-violet spectral range. Besides these absorptions water molecules show typical resonances at different wavelengths, leading to high absorption loss and almost $0 \%$ transmittance (see for example [8]). But fortunately the atmosphere shows high transmittance of $70 \% \leq \tau(L) \leq 90 \%$ at several wavelengths, allowing laser optical transmission. Some typical wavelengths of $800-850$ $\mathrm{nm}, 1064 \mathrm{~nm}$, or $1550 \mathrm{~nm}$ can be used for deep space optical communications.

\subsection{Pointing errors, spacecraft jitter, and point ahead errors}

Other critical effects, like pointing errors, spacecraft jitter, and point ahead errors, are not discussed explicitly in this paper, but can be implicitly considered by the pointing efficiency $\eta_{P}$ and spacecraft transmitter efficiency $\eta_{T}$ for link budget estimation.

\subsection{Turbulence, scintillation, and beam wander}

Besides Rayleigh scattering and Mie scattering at the molecular structures in the atmosphere, there are also turbulences caused by the motion of the atmosphere and clouds, with dimensions of a few millimeters to eddies of some meters. All these turbulences cause a stochastical fluctuation of the refractive index of the atmosphere, therefore phase shifts and refraction. The fluctuation of the refractive index of the eddies with sizes $l_{0}$ of few millimeters is called small-scale scintillation, whereas the large-scale fluctuation $L_{0}$ of the refractive index leads to refraction of the beams in a stochastical manner, so that the beam changes the propagation direction back and forth. This effect is also called beam wander. Both small-scale and large-scale scintillation lead to timely stochastical variation of the beam irradiance or the received power. This has to be taken into account.

The turbulences can be described by the Kolmogorov theory of turbulences (see for example [8]) and characterized by the so-called structure parameter of the refractive index fluctuation $C_{n}^{2}$ in $\left[\mathrm{m}^{-2 / 3}\right]$ which depends on the height above the sea level $h$ in $[\mathrm{m}]$, the wind velocity $v$ in $[\mathrm{m} / \mathrm{s}]$, the temperature $T(\mathbf{R})$ in $[\mathrm{K}]$ and the pressure $P(\mathbf{R})$ in [mbar], where $\mathbf{R}$ is the vector pointing from the coordinate center to the position in the atmosphere under consideration. The normalized refractive index for optical waves can be described by the following equation with the units for $v$ in $[\mathrm{m} / \mathrm{s}]$ and $h$ in $[\mathrm{m}]$ as well as $\lambda$ in $[\mu \mathrm{m}][8]$ with the second term describing the fluctuation or scintillation effect:

$n(\mathbf{R})=1+77.6 \cdot 10^{-6} \cdot\left(1+7.52 \cdot 10^{-3} \cdot \lambda^{-2}\right) \cdot \frac{P(\mathbf{R})}{T(\mathbf{R})}$

For $\lambda=0.5 \mu \mathrm{m}$ and $\lambda=1.55 \mu \mathrm{m}$ the refractive index fluctuation will be

$n(\mathbf{R})=1+79.9 \cdot 10^{-6} \cdot \frac{P(\mathbf{R})}{T(\mathbf{R})}$

$n(\mathbf{R})=1+77.8 \cdot 10^{-6} \cdot \frac{P(\mathbf{R})}{T(\mathbf{R})}$

The widely used wavelengths are $0.84 \mu \mathrm{m}, 1.064 \mu \mathrm{m}$ and $1.55 \mu \mathrm{m}$. In this project we assume one single formula (9) for the complete wavelength range for the optical laser communications $\lambda=0.5-1.55 \mu \mathrm{m}$ with sufficient accuracy, also given by [9], which is exact for $1.0 \mu \mathrm{m}$ :

$n(\mathbf{R})=1+79 \cdot 10^{-6} \cdot \frac{P(\mathbf{R})}{T(\mathbf{R})}$

All the parameters change permanently depending on wind velocity and height, and can be calculated using an empirical formula, i.e. Hufnagel-Valley $\mathrm{H}-\mathrm{V}$-model with $A=1.7 \cdot 10^{-14} \mathrm{~m}^{-2 / 3}$ for a typical wind velocity $v=21 \mathrm{~km} / \mathrm{h}$ $\approx 5.83 \mathrm{~m} / \mathrm{s}[8]$

$$
\begin{aligned}
C_{n}^{2}(h, v)= & 0.00594 \cdot\left(\frac{v}{27}\right)^{2} \cdot\left(10^{-5} h\right)^{10} \cdot \exp \left(\frac{-h}{1000}\right) \\
& +2.7 \cdot 10^{-16} \cdot \exp \left(\frac{-h}{1500}\right)+A \cdot \exp \left(\frac{-h}{100}\right)
\end{aligned}
$$

The lower the wind velocity, the higher the altitude, the lower the value of $C_{n}^{2}$ will be. Especially for the case if the altitude of the ground station is chosen higher, rather than at the ground level, so that the effective distance between the spacecraft and the ground station becomes significantly shorter. The $C_{n}^{2}$ values can be significantly reduced also by considering the elevation angle.

There are many software programs for H-V-Model calculation or a graphical diagram [9] can be used to find the typical values $C_{n}^{2} \approx 10^{-14}-10^{-13} \mathrm{~m}^{-2 / 3}$ at ground or sea level, also called strong turbulence; $C_{n}^{2} \approx 10^{-16} \mathrm{~m}^{-2 / 3}$ at height of about $1 \mathrm{~km}$ above the sea level; $C_{n}^{2} \approx 10^{-17} \mathrm{~m}^{-2 / 3}$ at $h=3 \mathrm{~km}$ above the sea level, with wind velocity $v=$ $21 \mathrm{~km} / \mathrm{h}$, rather weak turbulence; $C_{n}^{2}<10^{-18}-10^{-17} \mathrm{~m}^{-2 / 3}$ at $h=3-10 \mathrm{~km}$ above the sea level, with weak or no wind; $C_{n}^{2}<10^{-19}-10^{-18} \mathrm{~m}^{-2 / 3}$ at $h>10 \mathrm{~km}$ above the sea level. The near earth troposphere changes gradually 
from approximately $6-15 \mathrm{~km}$, depending on the latitude, to tropopause.

The Rytov variance for spherical waves can be used to estimate the standard deviation of the irradiance or the field amplitude with the units for $k$ in $\left[\mathrm{m}^{-1}\right]$ and $L$ in [m]

$\sigma_{I}^{2}(L)=0.5 \cdot C_{n}^{2} \cdot k^{7 / 6} \cdot L^{11 / 6}$

\section{Simulation of various scenarios for FSOC and DSOC}

\subsection{Links between GEO satellites ARTEMIS and earth ground station}

ESA developed optical terminals for high data rate free space laser communication via intersatellite (ISL) and interorbit links (IOL) within the frame of the Data Relay and Technology Mission (DRTM) program demonstrating for the first time that the stringent pointing, acquisition, and tracking requirements with infrared optical wavelengths is feasible [6]. For the purpose of in-orbit check-out of such payloads, ESA has also established an Optical Ground Station (OGS) at the Observatorio del Teide at Izaña, Tenerife, Spain. Tenerife has the ideal condition with extremely low atmospheric loss and scintillation. The OGS communicates with the optical laser system OPALE onboard ARTEMIS. The measurement campaign was conducted from 2003 to 2004 and valuable measurement data were collected. Using these measurement data, the link budget calculation can be verified and also the scintillation estimation can be validated.

The basic downlink and uplink parameters are listed in Tables 1 and 2. The simulation results for uplink and downlink, with consideration of the scintillation effect, are compared with the ARTEMIS measurement data in Table 3. For the simulation, first the link budget for irradiance $I$, which represents the mean value of the stochastical intensity variations due to the scintillation effect and received power $P$, is estimated. Then the index of the refractive structure

Table 1 Downlink ARTEMIS OPALE to OGS

\begin{tabular}{ll}
\hline ARTEMIS OPALE-OGS & $38,018 \mathrm{~km}$ \\
\hline TX power & $15 \mathrm{~mW}$ \\
TX laser diode wavelength & $815 \mathrm{~nm}$ \\
TX efficiency & 0.7 \\
TX aperture & $10 \mathrm{~cm}$ or $20 \mathrm{~cm}$ \\
Atmospheric transmission & 0.73 \\
RX aperture efficiency with filter & 0.16 \\
RX aperture diameter & $1 \mathrm{~m}$ \\
Blue sky brightness, $3 \mathrm{~nm}$ filter & $6 \mathrm{nW} / \mathrm{m}^{2}$ \\
\hline
\end{tabular}

Table 2 Uplink OGS to ARTEMIS OPALE

\begin{tabular}{ll}
\hline OGS-ARTEMIS OPALE & $38,018 \mathrm{~km}$ \\
\hline TX power $4 \times$ & $75 \mathrm{~mW}$ \\
TX laser diode wavelength & $847 \mathrm{~nm}$ \\
TX efficiency & 0.7 \\
TX aperture $4 \times$ & $10 \mathrm{~cm}$ \\
Atmospheric transmission & 0.8 \\
RX aperture efficiency with filter & 0.16 \\
RX aperture diameter & $0.1 \mathrm{~m}$ \\
Earth background brightness, $5 \mathrm{~nm}$ filter & $43.66 \mathrm{nW} / \mathrm{m}^{2}$ \\
\hline
\end{tabular}

parameter $C_{n}^{2}$, which will be influenced by wind velocity, temperature, and atmospheric pressure jointly, is calculated. At the end, the Rytov variance $\sigma_{I}^{2}$ as stochastical variation of the intensity, which corresponds to the standard variation of the stochastical process with a sampling period of $20 \mathrm{~ms}$, will be derived from $C_{n}^{2}$. The results were combined and compared with the ARTEMIS measurement data. $C_{n}^{2}$ is chosen to be $5 \cdot 10^{-18} \mathrm{~m}^{-2 / 3}$ for both downlink and uplink, to achieve the best match, since the site of the OGS in Tenerife is on top of the mountain with $\mathrm{h}=2393 \mathrm{~m}$ above the sea level and the measurements were performed during different daytimes. Since there are no contemporaneous weather measurement data like wind velocity, atmospheric pressure, and temperature available, we could not consider the possibly changing weather parameters dynamically and instead have chosen only the above mentioned effective $C_{n}^{2}$ parameter with which all simulation results match pretty well with the measurement data. The comparison shows that the simulation method can be used for the further link budget investigations and project design with sufficient accuracy.

The simulation gives the mean irradiance based on spherical beam propagation and/or Gaussian beam propagation with identical results. The irradiance varies with time, described by the Rytov variance $\sigma_{I}^{2}$. For the simulation, the time series have been matched to the measurement data scenario. The irradiance variation is obtained from the standard deviation $\sigma_{I}$ and is simulated with Gaussian distributed random numbers for subsequent data points.

The measurement of the downlink laser transmission experiment started on May 6, 2003 at 22:15 h and stopped after 914 seconds. During the measurement of the uplink laser transmission experiment starting on May 24, 2003 at 17:00 $\mathrm{h}$, after the link acquisition, the transmission bit error rate (BER) analysis started 278 seconds later. The transmitter switched the uplink lasers gradually from 4 lasers to 3 lasers 898 seconds after the start, then to 2 lasers after 1348 seconds, and finally to 1 laser after 1588 seconds. The measurement was stopped after 1798 seconds. The differences between simulation and measurement results should 
Fig. 1 Measurement results for Artemis downlink [4]
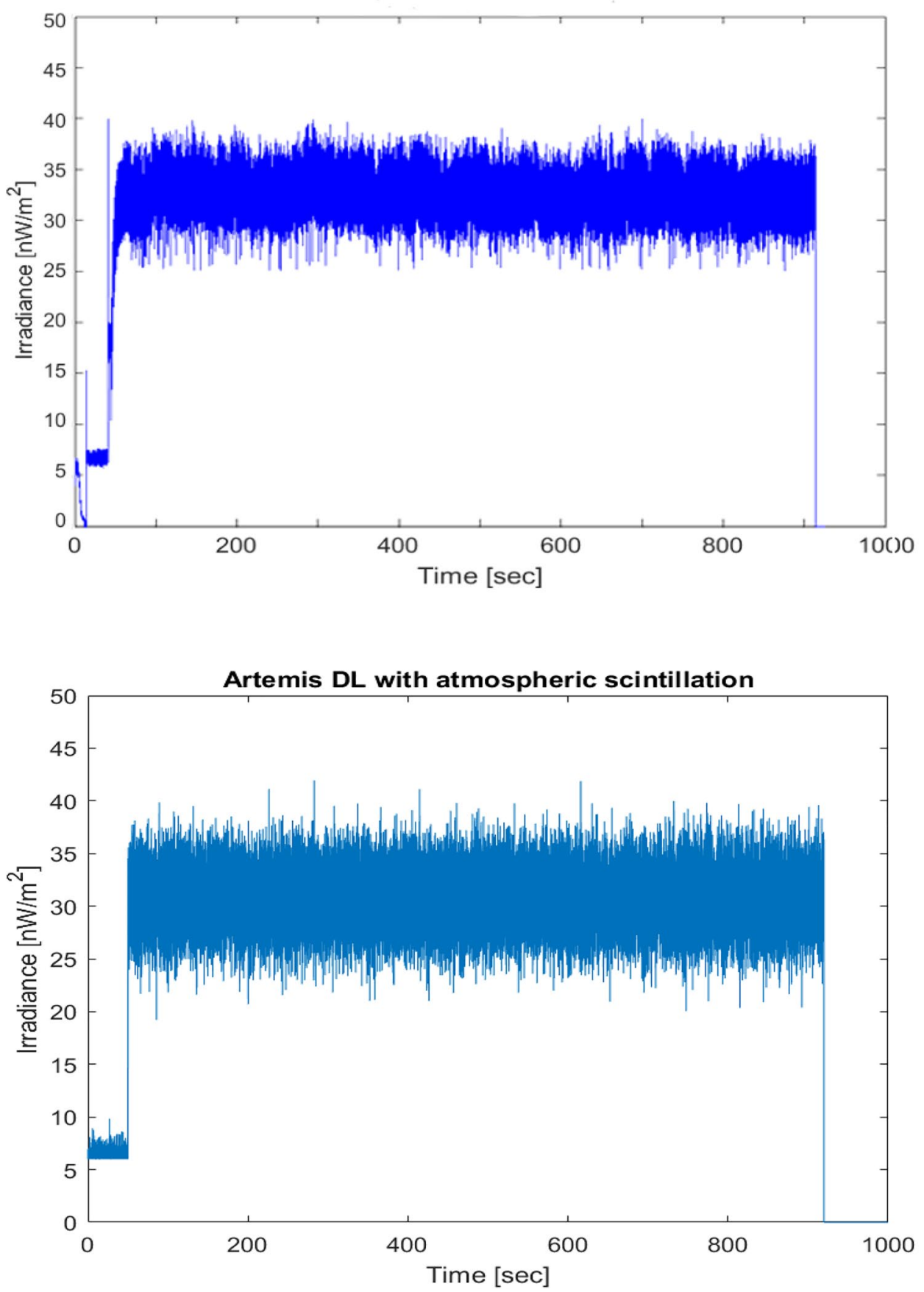

Fig. 2 Simulation results for ARTEMIS downlink be further analyzed. Figure 1 shows the measured downlink, Fig. 2 shows the simulated downlink, Fig. 3 shows the measured uplink, and Fig. 4 shows the simulated uplink. As discussed before, due to the missing simultaneous weather measurement data which could lead to significant changes of the $C_{n}^{2}$ values, we assumed an effective constant $C_{n}^{2}$ value for the complete measurement duration. This explains also the rather smaller difference between the simulation results and measurement results at the beginning with 4 laser beams compared with the case with only 1 laser beam at the end, possibly due to the temperature change and/or wind velocity changes during the measurement.

\subsection{Links between Lagrange $L 5$ and earth ground station}

ESA plans to embark the Deep Space Optical Communications System (DOCS) on the Space WEather (SWE) mission to the Sun-Earth Lagrange point L5 in the frame of its Space Situational Awareness (SSA) program [7]. DOCS 
Fig. 3 Measurement results for Artemis uplink [4]
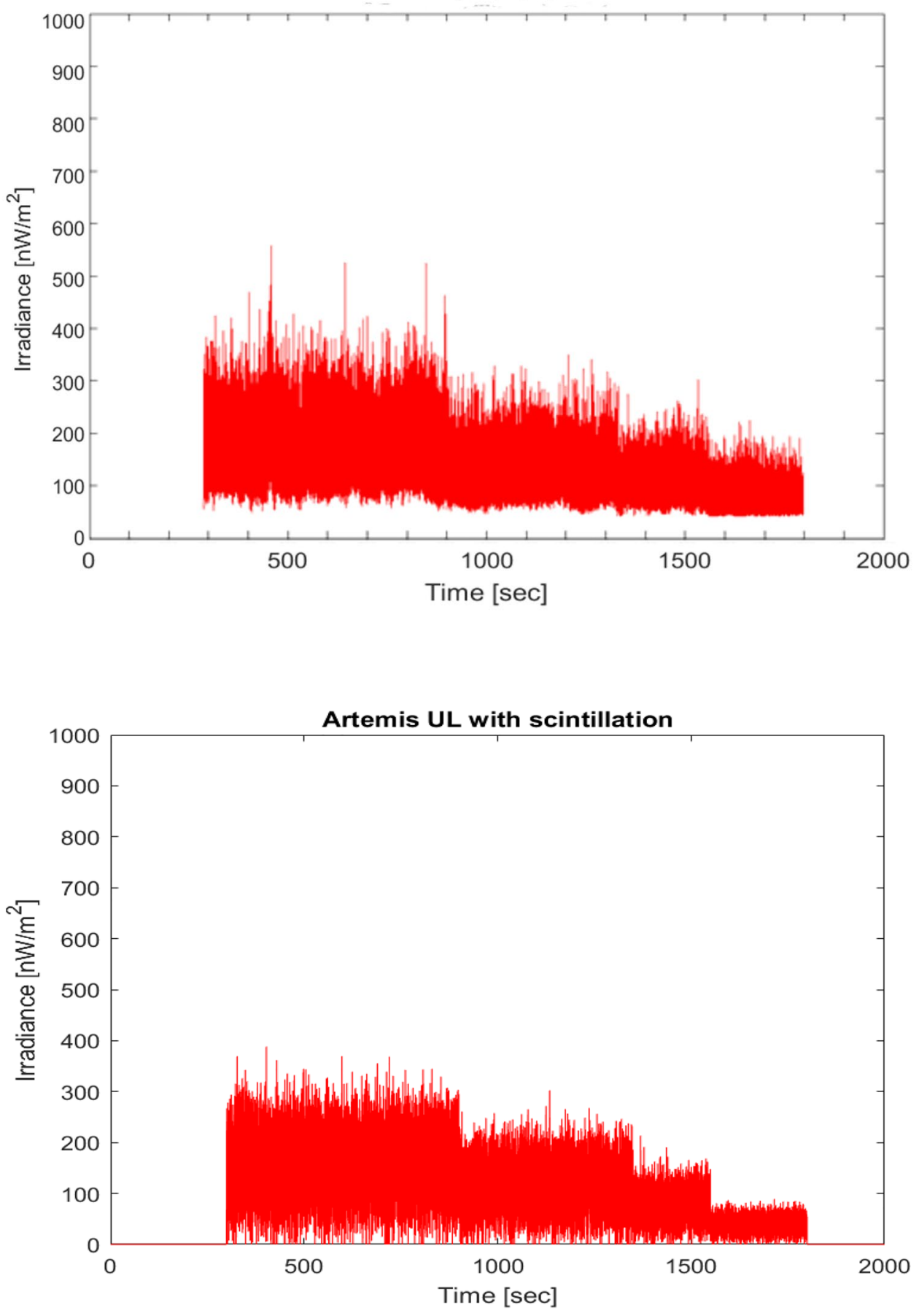

Fig. 4 Simulation results for ARTEMIS uplink
Table 3 Comparison of simulation (DL, UL) with measurement data [4]

\begin{tabular}{lll}
\hline & mean irradiance $I$ & scintillation $\sigma_{I}$ \\
\hline Artemis measurement DL & $33.76 \mathrm{nW} / \mathrm{m}^{2}$ & $2.29 \mathrm{nW} / \mathrm{m}^{2}$ \\
Simulation DL & $32.00 \mathrm{nW} / \mathrm{m}^{2}$ & $1.30 \mathrm{nW} / \mathrm{m}^{2}$ \\
Artemis measurement UL & $122.84 \mathrm{nW} / \mathrm{m}^{2}$ & $43.81 \mathrm{nW} / \mathrm{m}^{2}$ \\
Simulation UL & $103.69 \mathrm{nW} / \mathrm{m}^{2}$ & $29.22 \mathrm{nW} / \mathrm{m}^{2}$ \\
\hline
\end{tabular}

is an in-orbit technology demonstration that also serves a scientific objective, namely the transfer of high-resolution solar imagery. The characteristics of the SWE L5 mission provide substantial advantages for deep space optical communications, because the equidistant triangular orbital geometry between Sun, Earth, and L5 (all three distances are equal to $1 \mathrm{AU} \approx 150$ million $\mathrm{km}$ ) ensures that the sun is always separated by 60 degrees from both the space and the 
ground terminal. This allows for very efficient solar straylight shielding and thermal management. It also reduces the pointing requirements of the DOCS Space Terminal (DST). A coarse pointing mechanism is not required, nor is a pointahead assembly. Also in this project, the OGS in Tenerife, Spain will play an important role. It is called DOCS Ground Terminal (DGT).

The system parameters for downlink and uplink are listed in Tables 4 and 5. The comparison with the designed link budget of [7] is shown in Table 6. The independent simulation results in this work match with the link budget calculation pretty well. In DSOC, besides the atmospheric scintillation also other effects, like solar plasma or sun irradiance, would affect the laser transmission. Once the first DSOC measurement data are available, for example from the Psyche mission for distances up to $3 \mathrm{AU}$, it will be illuminating to investigate the scintillation effects to further improve our simulation methods. Based on the results achieved up to now, we are optimistic that the simulation methods documented in this paper can be further developed to more completely characterize optical communications for deep space missions at interplanetary distances.

\section{Further considerations and steps for the performance analysis and deep space mission planning}

As discussed above, it would be meaningful to collect laser link performance measurement data along with the critical weather data like wind velocity, temperature and pressure in the atmosphere in the future mission experiments, to better understand the temporal changes of the mean irradiance and the scintillation effects.

For interplanetary deep space missions with distance of 1-3 AU, when the photons are extremely starved, it is

Table 4 Downlink DOCS DST to DGT

\begin{tabular}{ll}
\hline DOCS DST-DGT & $\begin{array}{l}1 \mathrm{AU} \approx \\
150,000,000 \\
\mathrm{~km}\end{array}$ \\
\hline TX power & $15 \mathrm{~mW}$ \\
TX laser diode wavelength & $1550 \mathrm{~nm}$ \\
TX efficiency & 0.7 \\
TX aperture & $20 \mathrm{~cm}$ \\
Atmospheric transmission & 0.73 \\
RX aperture efficiency with filter & 0.5 \\
RX aperture diameter & $1 \mathrm{~m}$ or $4 \mathrm{~m}$ \\
Blue sky brightness, $3 \mathrm{~nm}$ filter & $0.5 \mathrm{pW}$ \\
\hline
\end{tabular}

Table 5 Uplink DOCS DGT to DST

\begin{tabular}{ll}
\hline DOCS DGT-DST & $\begin{array}{l}1 \mathrm{AU} \approx \\
150,000,000 \\
\mathrm{~km}\end{array}$ \\
\hline TX power $4 \times$ & $600 \mathrm{~W}$ \\
TX laser diode wavelength & $1064 \mathrm{~nm}$ \\
TX efficiency & 0.7 \\
TX aperture $4 \times$ & $10 \mathrm{~cm}$ \\
Atmospheric transmission & 0.8 \\
RX aperture efficiency with $5 \mathrm{~nm}$ filter & 0.7 \\
RX aperture diameter & $20 \mathrm{~cm}$ \\
Earth background, $5 \mathrm{~nm}$ filter & $0.8 \mathrm{pW}$ \\
\hline
\end{tabular}

of great importance to consider the penalty of the daytime measurement versus nighttime measurement, since the blue sky background and Earth background noise could significantly affect the measurement. These effects must be considered and utilized in the future DSOC investigations correspondingly.

Another huge factor for mission planning is also the socalled SEP (Sun-Earth Probe angle), i.e. the closeness of the spacecraft to the Sun, as seen on the sky. Just like the radio frequency propagation will be disturbed by the solar plasma, the optical wave propagation will be also affected by the infrared radiation of the Sun significantly, leading to performance degradation. This effect must also be taken into account in future deep space mission planning.

For interplanetary DSOC with starved photons at the receivers, single photon counting APD is indispensable. Proper PPM orders have to be chosen, and the deadtime of the single photon counting APD must be taken into account.

The site dependencies are very influential to mission planners for designing DSOC systems. The weather conditions like wind velocity, clouds and elevation angles are crucial factors. The above mentioned simulation method for the link budget analysis and the scintillation estimation will be combined, further improved in a proper way for the analysis of the future missions.

As documented in this paper, we have verified our simulation methods by comparison with existing mission planning

Table 6 Comparison of the designed link budget [7] and simulation in this work

\begin{tabular}{llll}
\hline & $\begin{array}{l}\text { Downlink 4 m } \\
\text { receiver }\end{array}$ & $\begin{array}{l}\text { Downlink 1 m } \\
\text { receiver }\end{array}$ & Uplink \\
\hline DOCS Design & $9.30 \mathrm{pW}$ & $0.60 \mathrm{pW}$ & $8.50 \mathrm{pW}$ \\
This work & $9.43 \mathrm{pW}$ & $0.59 \mathrm{pW}$ & $9.02 \mathrm{pW}$ \\
\hline
\end{tabular}


design data DOCS L5 and ARTEMIS OGS OPALE measurement results, under somewhat constrained or limited conditions. With these successful verifications, in the future, we plan to further improve the simulation algorithms to more completely represent additional factors that impact the performance of deep space optical communications.

\section{Conclusions}

The simulation methodology for free space optical communications (FSOC) and deep space optical communications (DSOC) is presented. By using the simulation algorithm two scenarios for a link a) between the near earth GEO satellite ARTEMIS, b) between the Lagrange point L5 and the optical ground station OGS have been investigated. Especially, the valuable measurement data of ESA's ARTEMIS SILEX project are used to verify not only the link budget calculation, but also the critical scintillation estimation with feasible parameters. Since the structure function for the refractive index is changing over the large distance, also varying due to changes of temperature, pressure, wind, azimuth, and elevation angles, one can not find an analytical set of parameters which are valid for all scenarios. The comparison shows that the simulation models for the link budget and the scintillation estimation are feasible for the investigations of free space and deep space optical communications. This investigation helps to find a reliable empirical data set for each project with typical constellations, daytimes, and typical weather conditions. In the near future we will be working on link performance analyses for downlink and uplink between Earth ground station and lunar orbits, and between Earth ground station and other planets or asteroids by using realistic design parameters.

Acknowledgements Shun-Ping Chen would like to thank Darmstadt University of Applied Sciences h-da and European Space Agency ESA/ ESOC for the valuable opportunity to be a visiting scientist at ESA/ ESOC for one year, especially OPS-GSO Section Head Dr. Clemens Heese for his strong support and many helpful discussions during this project. The author also appreciates the continuously good cooperation and many fruitful discussions with Prof. Dr. Heinz Schmiedel about Microwave Engineering and Optical Communications. The author would also like to thank the anonymous reviewers for carefully reading the paper, for many detailed valuable comments and helpful suggestions for improvement.

Funding Open Access funding enabled and organized by Projekt DEAL.
Open Access This article is licensed under a Creative Commons Attribution 4.0 International License, which permits use, sharing, adaptation, distribution and reproduction in any medium or format, as long as you give appropriate credit to the original author(s) and the source, provide a link to the Creative Commons licence, and indicate if changes were made. The images or other third party material in this article are included in the article's Creative Commons licence, unless indicated otherwise in a credit line to the material. If material is not included in the article's Creative Commons licence and your intended use is not permitted by statutory regulation or exceeds the permitted use, you will need to obtain permission directly from the copyright holder. To view a copy of this licence, visit http://creativecommons.org/licenses/by/4.0/.

\section{References}

1. Hemmati, H., Biswas, A., Djordjevic, I.B.: Deep Space Optical Communications: Future Perspectives and Applications. Proceedings of IEEE 99(11),(November 2011)

2. Sans, M., Sodnik, Z., Zayer, I., Daddato, R.: ' 'Design of the ESA Optical Ground Station for Participation in LLCD", Proc. International Conference on Space Optical Systems and Applications (ICSOS) 2012, 3-1, Ajaccio, Corsica, France, October 9-12, 2012

3. Sodnik, Z., Czichy, R.: "Design Data Summary of the ESA Optical Ground Station for In-Orbit Check-Out of Laser Communication Payloads and for the Observation and Registration of Space Debris". ESA Specifications for the Artemis OGS OPALE project. Ref.: TEC-MMO/267/ZS. May 2, 2005

4. Internal ESA Artemis OGS OPALE Measurement Results. 2003-2004

5. Mendenhall, J., Candell, L., Hopman, P., Zogbi, G., Boroson, D., Caplan, D., Digenis, C., Hearn, D., Shoup, R.: "Design of an Optical Photon Counting Array Receiver System for Deep-Space Communications", Proceedings of IEEE, vol. 95, Nr. 10, October 2007

6. Sodnik, Z., Smit, H., Sans, M., Giggenbach, D., Becker, P., Mata-Calvo, R., Fuchs, C., Zayer, I., Anucara, M.L., Schulz, K., Widmer, J., Arnold, F., Mosberger, M., Alonso, A., Montilla, I.: "Results from a Lunar Laser Communication Experiment between NASA's LADEE Satellite and ESA's Optical Ground Station", Proc. International Conference on Space Optical Systems and Applications (ICSOS) 2014, S2-1, Kobe, Japan, May 7-9, 2014

7. Sodnik, Z., Heese, C., Arapoglou, P.-D., Schulz, K.-J., Zayer, I., Daddato, R., Kraft, S.: "Deep-Space Optical Communication System (DOCS) for ESA's Space Weather Mission to Lagrange Orbit L5". IEEE International Conference on Space Optical Systems and Applications (ICSOS), November 14-16, 2017

8. Andrews, L.C., Phillips, R.L., Hopen, C.Y.: Laser Beam Propagation through Random Media. SPIE Press, Bellingham, Washington USA (2005)

9. Andrews, L.C., Phillips, R.L., Hopen, C.Y.: Laser Beam Scintillation with Applications. SPIE Press, Bellingham, Washington USA (2001) 